https://helda.helsinki.fi

Response univariance in bull-frog rods with two visual pigments

Firsov, M.L.

Elsevier

1994

Vision Research. 1994. 34: 839-847

http://hdl.handle.net/1975/933

http://dx.doi.org/doi:10.1016/0042-6989(94)90034-5

Downloaded from Helda, University of Helsinki institutional repository.

This is an electronic reprint of the original article.

This reprint may differ from the original in pagination and typographic detail.

Please cite the original version. 


\title{
Response Univariance in Bull-Frog Rods with Two Visual Pigments
}

\author{
MICHAEL L. FIRSOV, * VICTOR I. GOVARDOVSKII,* KRISTIAN DONNER $\dagger$
}

Received 4 May 1993; in revised form 16 August 1993

\begin{abstract}
Rods in the bull-frog retina contain varying proportions of rhodopsin $\left(\lambda_{\max }=502 \mathrm{~nm}\right)$ and porphyropsin $\left(\lambda_{\max }=527 \mathrm{~nm}\right)$ in a dorso-ventral gradient from the porphyropsin-rich dorsal rim to the virtually pure rhodopsin fields of the central and ventral retina. We investigated if quantal excitations in the same rod are different depending on whether they are initiated by isomerization of a rhodopsin or a porphyropsin molecule. Current photoresponses were recorded from dark-adapted rods by sucking the outer segment into a recording pipette. The relation between pigment composition and spectral sensitivity was established by comparison with microspectrophotometrically measured absorbance spectra of rods from the same neighbourhood. Rods with suitable porphyropsin: rhodopsin mixtures (ideally between 1:4 and 1:2) were stimulated with flashes of red $(608 \mathrm{~nm})$ and blue $(465 \mathrm{~nm})$ light, whereby the red light will isomerize porphyropsin much more often than rhodopsin, and the reverse will be true of the blue light. The amplitude and shape of the single-photon response were found to be identical for the "red" and "blue" flash series to within measurement error (ca 10\%). This indicates that the quantal responses initiated by the two pigments are identical.
\end{abstract}

$\underline{\text { Phototransduction Rhodopsin Porphyropsin Retinal Chromophore Bull-frog }}$

\section{INTRODUCTION}

Response univariance means that the shape and amplitude of the photoreceptor response depends only on the number of effectively absorbed photons but not on their wavelength. The "principle of univariance" (Naka \& Rushton, 1966) has been found to hold generally, as is expected if a photon irrespective of its energy produces the same activated form of the visual pigment molecule. In the case of photoreceptors with mixed pigment content, it is not obvious that this should be the case. In particular, rods and cones in many species of fish and amphibians contain mixtures of pigments that differ in chromophoric group, based on either vitamin $\mathrm{A}_{1}$ (retinal) or vitamin $A_{2}$ (3-dehydroretinal), and thereby in spectral sensitivity (Bridges, 1970; Reuter, White \& Wald, 1971; Semple-Rowland \& Goldstein, 1981; Crescitelli, 1958, 1990; Donner, Firsov \& Govardovskii, 1990).

The key product in the visual pigment bleaching sequence is metarhodopsin II (MII) which sets off the excitation cascade. Both the amplitude and the kinetics of the photoresponse depend on the kinetics and efficiency of the interaction between MII and transducin as well as the kinetics of MII inactivation by phosphoryl-

*Institute of Evolutionary Physiology and Biochemistry, Russian Academy of Sciences, St Petersburg, Russia.

$\dagger$ Department of Zoology, P.O. Box 17, FIN-00014, University of Helsinki, Helsinki, Finland. ation and arrestin binding (reviewed by Pugh \& Lamb, 1990). In MII the chromophore is still bound to its original site, so the nature of the chromophore might influence the properties of the molecule and hence the photoresponse. Recently, Corson, Derguini, Nakanishi, Crouch, Macnichol and Cornwall (1991) have reported that substitution of the native chromophore by the artificial analogue 9-desmethyl retinal makes response shape dependent on wavelength in salamander rods. Conceivably, the different native chromophores of the rod pigments rhodopsin (retinal) and porphyropsin (3-dehydroretinal) could also be associated with such differences.

In this work we investigate the possibility that single-quantum responses (SQRs) initiated by rhodopsin and porphyropsin in the same cell could be different, using rods of the bull-frog (Rana catesbeiana) containing various proportions of the two pigments. Porphyropsin absorbs long wavelengths better than rhodopsin, so (partially) selective excitation can be achieved by use of short-wavelength ("blue", here $465 \mathrm{~nm}$ ) or long-wavelength ("red", here $608 \mathrm{~nm}$ ) stimulus flashes. Current photoresponses were recorded from single rods drawn into suction pipettes, and the amplitudes and kinetics of "blue" and "red" responses were determined. To within measurement error ( $c a$ $10 \%$ ), no differences were detected, which implies that rhodopsin- and porphyropsin-initiated SQRs are identical. 


\section{MATERIALS AND METHODS}

\section{Animals and preparation}

Adult bullfrogs, Rana catesbeiana, were obtained from KONS Scientific Co., Inc. (Germantown, Wis.) and kept on a $12: 12 \mathrm{hr}$ light-dark cycle at $12-14^{\circ} \mathrm{C}$ in basins with a white floor. These conditions favour the development of the dorsal porphyropsin field (Tsin \& Beatty, 1980). Prior to the experiment, frogs were dark-adapted overnight at room temperature. A frog was killed by decapitation and double-pithed, and the eyes were removed under dim red light. Small segments of the eyecup were excised from the desired region, usually midway between the horizontal meridian and the dorsal border (to obtain pieces of retina with mixed pigment content). The retina was isolated in Ringer solution containing (mM): $\mathrm{NaCl}, 90 ; \mathrm{KCl}, 2.5 ; \mathrm{MgSO}_{4}, 1.0 ; \mathrm{CaCl}_{2}, 1.0$; $\mathrm{NaHCO}_{3}, 5.0$; glucose, 10; HEPES, 10 (pH 7.5-7.7) and sodium aspartate, 10 (to eliminate possible synaptic feedback from other rods).

\section{Microspectrophotometry}

The pigment composition of a rod can be determined from its absorption spectrum. For about half of the rods studied electrophysiologically, other rods from the same small neighbourhood were subjected to microspectrophotometric examination as described by Govardovskii and Zueva (1988) and Donner et al. (1990). Averaged absorption spectra from 20-40 single rod outer segments in each case were computer-fitted with sums of rhodopsin and porphyropsin nomograms and related to the electrophysiological data.

In fitting nomograms we basically used the eighthorder polynomial expressions of Dawis (1981) for rhodopsin ( $\mathrm{A}_{1}$-based pigment) and porphyropsin $\left(\mathrm{A}_{2}-\right.$ based pigment). However, the rhodopsin curve was modified to give somewhat lower absorbance in the long-wavelength end (substituting Dawis' coefficients $b_{7}=-789.371$ and $b_{8}=-60749.2$ by $b_{7}=5211.534$ and $b_{8}=-95544.79$ ). The modified nomogram corresponds closely to the rhodopsin template published by Partridge and de Grip (1991). Further, as suggested to us by Maksimov (1988), $\left(\lambda_{\max } / \lambda-1\right)$ was used as parameter, which makes the curves shape-invariant on a relative frequency scale (Mansfield, 1985; MacNichol, 1986).

In a previous study (Donner et al., 1990), we assumed $\lambda_{\max }=501.5$ and $523 \mathrm{~nm}$ for bull-frog rhodopsin and porphyropsin (essentially following Reuter et al., 1971). Our present re-examination indicates that fits to mixed spectra improve perceptibly if a larger value for porphyropsin $\lambda_{\text {max }}$ is assumed. Here we use $\lambda_{\text {max }}=527 \mathrm{~nm}$, which is that expected for the porphyropsin paired with rhodopsin $\sin _{502}$ according to Dartnall's and Lythgoe's (1965) rule $\lambda_{1}=0.60 \lambda_{2}+186$. The discrepancy in relation to Reuter et al. (1971) may be due at least in part to the presence of non-negligible fractions of rhodopsin even in the dorsalmost porphyropsin extracts of these authors (their Fig. 2). This conjecture is supported by the fact that the estimated porphyropsin content of the most porphyropsin-rich dorsal rods was never higher than
$70-88 \%$ even when $\lambda_{\max }=523 \mathrm{~nm}$ was assumed (Donner et al., 1990). It is worth emphasizing, though, that our main conclusions do not depend on whether the porphyropsin peaks at 523 or $527 \mathrm{~nm}$. Assuming too high porphyropsin $\lambda_{\max }$ would mainly lead us to underestimate the porphyropsin percentage in cells with mixed pigment composition.

\section{Current recording}

The current of single dark-adapted rods protruding from the edge of small pieces of retina was recorded by the suction-pipette method as described earlier (Baylor, Lamb \& Yau, 1979a; Donner et al., 1990). The signal was amplified (bandpass $0.016-10 \mathrm{~Hz}$, in some cases $0-10 \mathrm{~Hz}$; four-pole active Butterworth filter), digitized at 20-100 msec interval and stored on hard disk. Low-pass filtering at $10 \mathrm{~Hz}$ does not significantly influence response waveforms, as they are dominated by much lower frequencies $(<1 \mathrm{~Hz}$; as exemplified in Figs 3 and 5 dim-flash responses typically peak around $1.5 \mathrm{sec}$ at room temperature).

\section{Stimulation}

The light came from two stabilized sources with interference and neutral density filters interposed in the beam. Stimuli were $50 \mathrm{msec}$ full-field flashes controlled by an electromagnetic shutter. The unattenuated intensity of light incident on the preparation was measured with a calibrated photomultiplier. A first estimate of average numbers of photoisomerizations per rod per flash was obtained from rod collecting areas and neutral filter densities (see Donner et al., 1990). The actual number of photoisomerizations during each particular stimulus session was estimated from response statistics. Let the mean amplitude of responses to repeated presentations of nominally identical flashes be $m$ and the variance $\sigma^{2}$. If variability is predominantly due to random variation of the numbers of photoisomerizations produced on individual trials, then (from Poisson statistics) the mean number of isomerizations per flash $(N)$ is

$$
N=m^{2} / \sigma^{2}
$$

\section{Selective excitation by different wavelengths}

Rods in the dorsalmost retina of the bull-frog contain predominantly porphyropsin whereas rods in the ventral retina contain almost pure rhodopsin (Reuter et al., 1971; Makino, Kuzuo \& Suzuki, 1983). Cells from the intermediate zone contain both pigments mixed in various proportions. Porphyropsin is more red-sensitive than rhodopsin, but the absorption spectra of the two pigments largely overlap. Therefore, selective absorption in one or the other can be only partly achieved, by use of wavelengths that are absorbed with different probabilities by the two pigments. Any pair of "short" $(<500 \mathrm{~nm})$ and "long" (>580 nm) wavelengths will basically do. In this work, 465 and $608 \mathrm{~nm}$ were used throughout. 
The proportions of quantal responses initiated by rhodopsin or porphyropsin depends critically on the proportions of the two pigments in the rod. For best differentiation, there should be more rhodopsin (to increase the rhodopsin advantage in absorbing shortwavelength light) but not too much (not to offset the advantage of porphyropsin in the long-wavelength region). Figure 1 summarizes how absorption in the two pigments depends on the relative porphyropsin content of the cell: (1) the thin continuous curve gives, for $465 \mathrm{~nm}$ photons, the ratio of absorptions in rhodopsin to those in porphyropsin molecules; (2) the dotted curve gives, for $608 \mathrm{~nm}$ photons, the ratio of absorptions in porphyropsin to those in rhodopsin molecules; (3) the bold continuous curve gives the ratio of 465 and $608 \mathrm{~nm}$ intensities that will produce equal total numbers of absorptions (normalized to 1 for pure rhodopsin). All are shown as functions of the fraction of porphyropsin of pigment total in the cell. For example, if there is $<10 \%$ porphyropsin, almost all $465 \mathrm{~nm}$ isomerizations will be in rhodopsin molecules. Such cells are not, however, advantageous for differentiating between the pigments, because even $608 \mathrm{~nm}$ photons will then isomerize rhodopsin more often than porphyropsin. Similarly, in cells with very high porphyropsin content ( $>60 \%$ ) almost all $608 \mathrm{~nm}$ isomerizations, but also the majority of $465 \mathrm{~nm}$ isomerizations will be in porphyropsin molecules. Optimal differentiation is achieved in an intermediate range (from 20 to $35 \%$ ). For example, with $35 \%$ porphyropsin and $65 \%$ rhodopsin, $465 \mathrm{~nm}$ photons will isomerize rhodopsin 2.5 times more often than porphyropsin, but $608 \mathrm{~nm}$ photons will isomerize porphyropsin 5 times more often than rhodopsin.
In the electrophysiological recordings, a rough estimate of the porphyropsin content was obtained by determining the ratio of 465 and $608 \mathrm{~nm}$ intensities needed to evoke the same response amplitude $\left(I_{465} / I_{608} \equiv\right.$ the ratio of sensitivities at 608 and $465 \mathrm{~nm}$, $\left.S_{608} / S_{465}\right)$. This ratio was scaled by setting the mean ratio of the most short-wavelength-sensitive rods from the ventral retina equal to 1 ; the normalized sensitivity ratio will be referred to as the $\mathrm{R} / \mathrm{B}$ ratio. The electrophysiologically determined $\mathrm{R} / \mathrm{B}$ ratio cannot of course $a$ priori be taken as a measure of the pigment composition: if the amplitudes of responses initiated by rhodopsin and porphyropsin are different, the ratio of intensities that produce equal response amplitudes will differ from the ratio of absorbances. However, once a correspondence between R/B and absorbance ratios has been established, pigment composition can be estimated from the R/B ratio alone, as was the case in about half of the rods analysed (i.e. for these cells no independent microspectrophotometric pigment determination is available).

\section{Protocol}

To avoid spurious differences due to sensitivity drift during long recording sessions, 465 and $608 \mathrm{~nm}$ flashes were interleaved in blocks of 20 flashes. Dim-flash blocks were alternated with occasional blocks of stronger flashes and with single saturating flashes to monitor changes in the saturating response amplitude. For the analysis, the recordings were chopped and reassembled into series of responses to flashes of one wavelength (see Fig. 2).

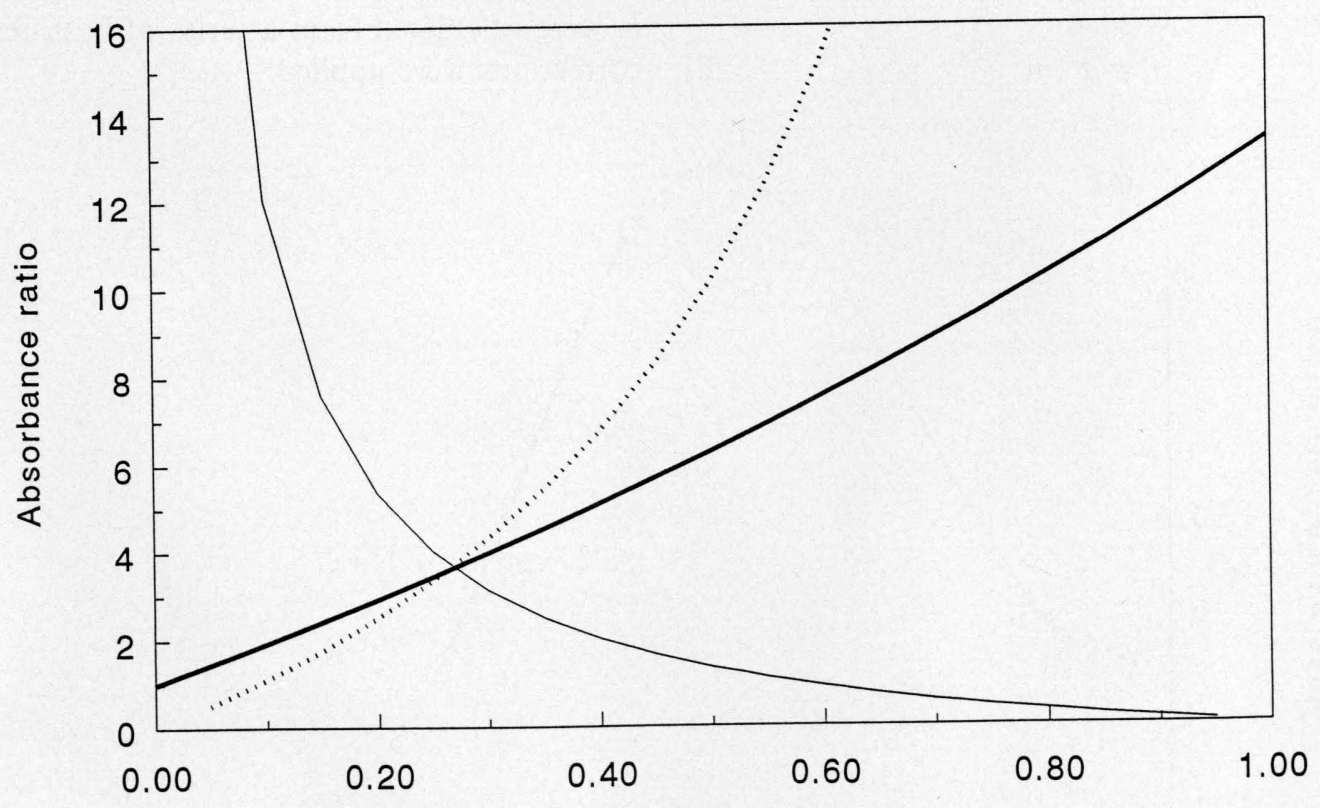

\section{Porphyropsin content}

FIGURE 1. Relative probabilities that blue $(465 \mathrm{~nm})$ and red $(608 \mathrm{~nm})$ photons are absorbed in rhodopsin or porphyropsin molecules, as functions of fraction porphyropsin in the cell. Thin solid line: ratio of rhodopsin absorptions to porphyropsin absorptions for $465 \mathrm{~nm}$ photons. Dotted line: ratio of porphyropsin absorptions to rhodopsin absorptions for $608 \mathrm{~nm}$ photons. Bold solid line: ratio of total absorption of $608 \mathrm{~nm}$ photons to total absorption of $465 \mathrm{~nm}$ photons, scaled so that the ratio $=1$ 


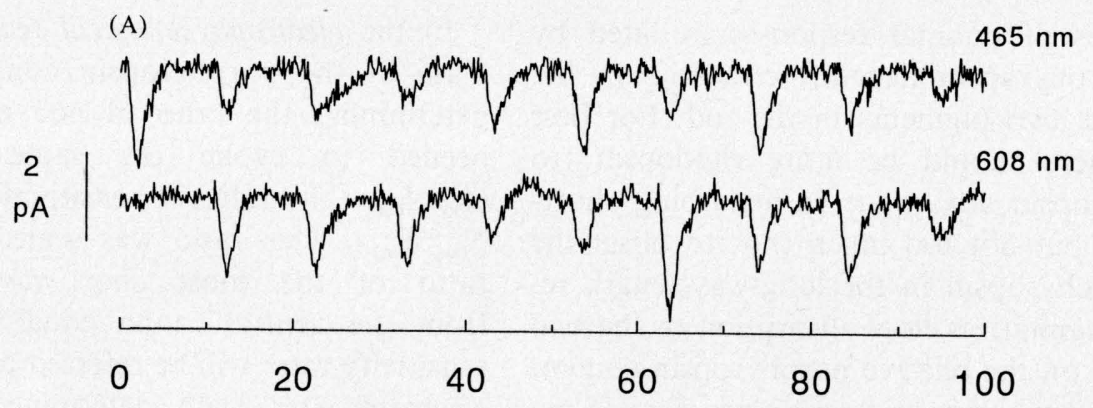

(B)

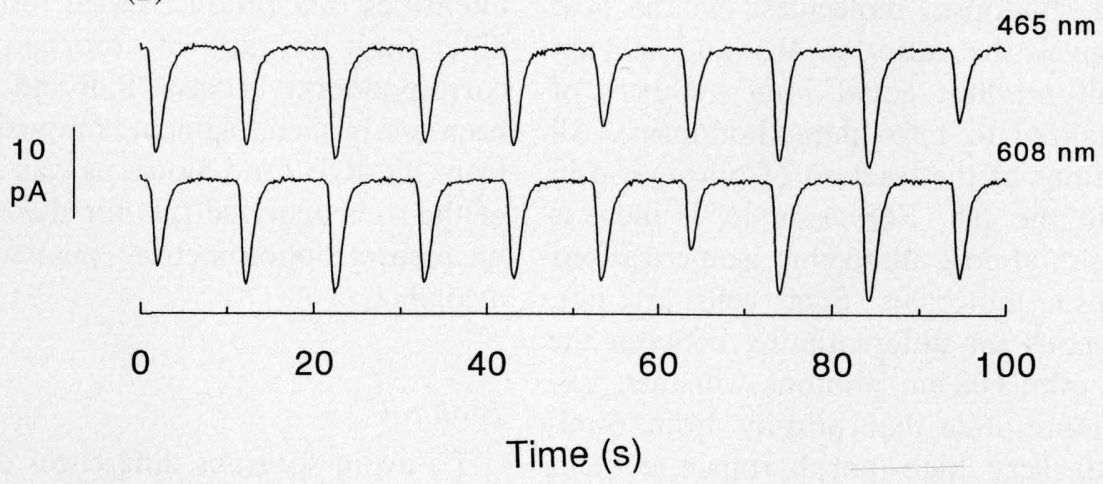

FIGURE 2. Series of individual flash responses from one rod from the dorsal retina: (A) ca 9 isomerizations per flash, (B) ca 40 isomerizations per flash. (The absorption spectrum from rods in the same neighbourhood is shown in Fig. 4 and averaged responses of the same cell in Fig. 5.)

\section{Estimation of SQR amplitude}

Denoting the SQR amplitude by $a_{q}$, it is selfevident that $m=N a_{q}$. Combining this with equation (1) we obtain the relation (cf. Baylor, Lamb \& Yau, 1979b)

$$
a_{q}=\sigma^{2} / m
$$

which gives an estimate of $a_{q}$ based on the assumption that the variation in response amplitudes is predominantly due to Poisson variation in the numbers of photoisomerizations produced on successive presentations of the same nominal flash intensity. Before $\sigma^{2}$ and $m$ were calculated from a series of flash responses, two corrections were applied.

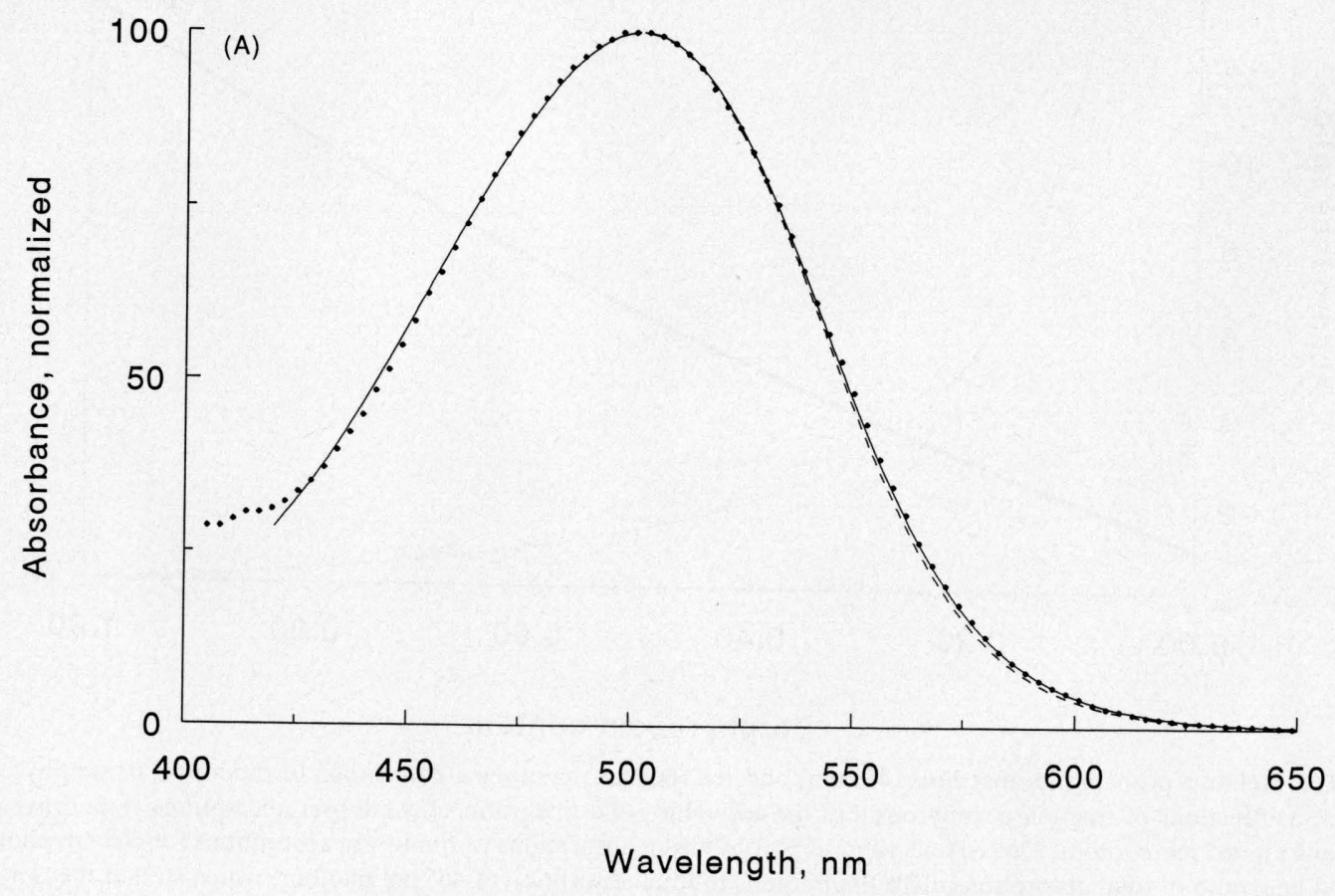

FIGURE 3(A). Caption on facing page. 
(B)

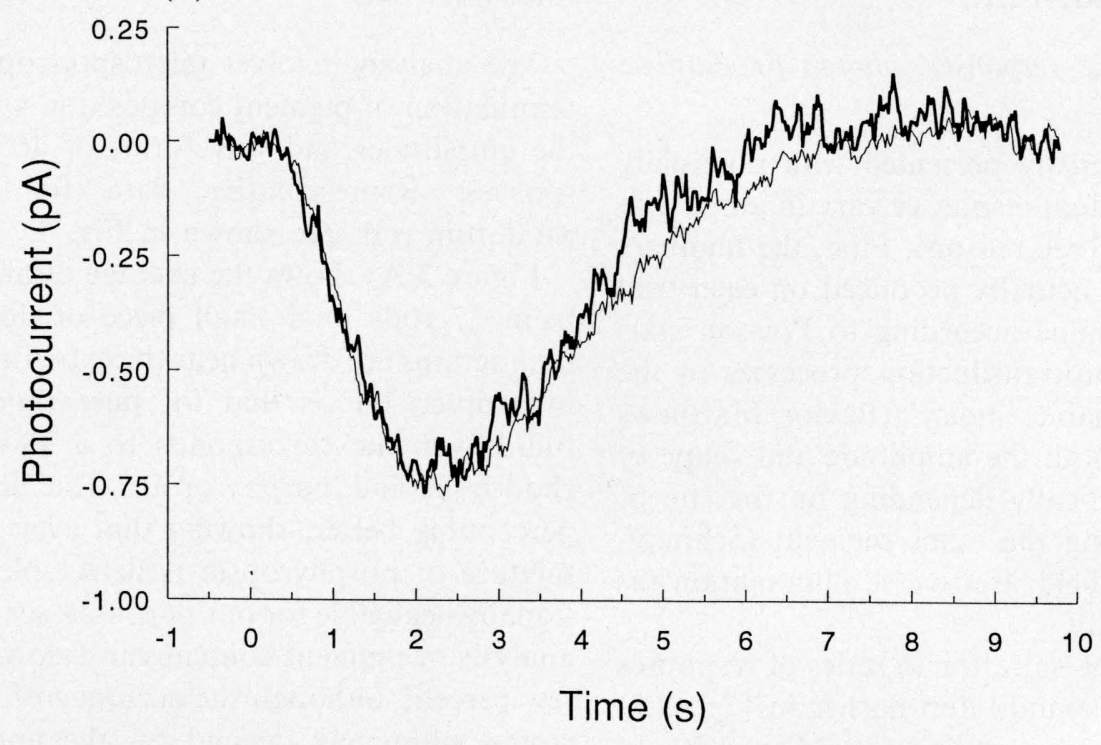

(C)

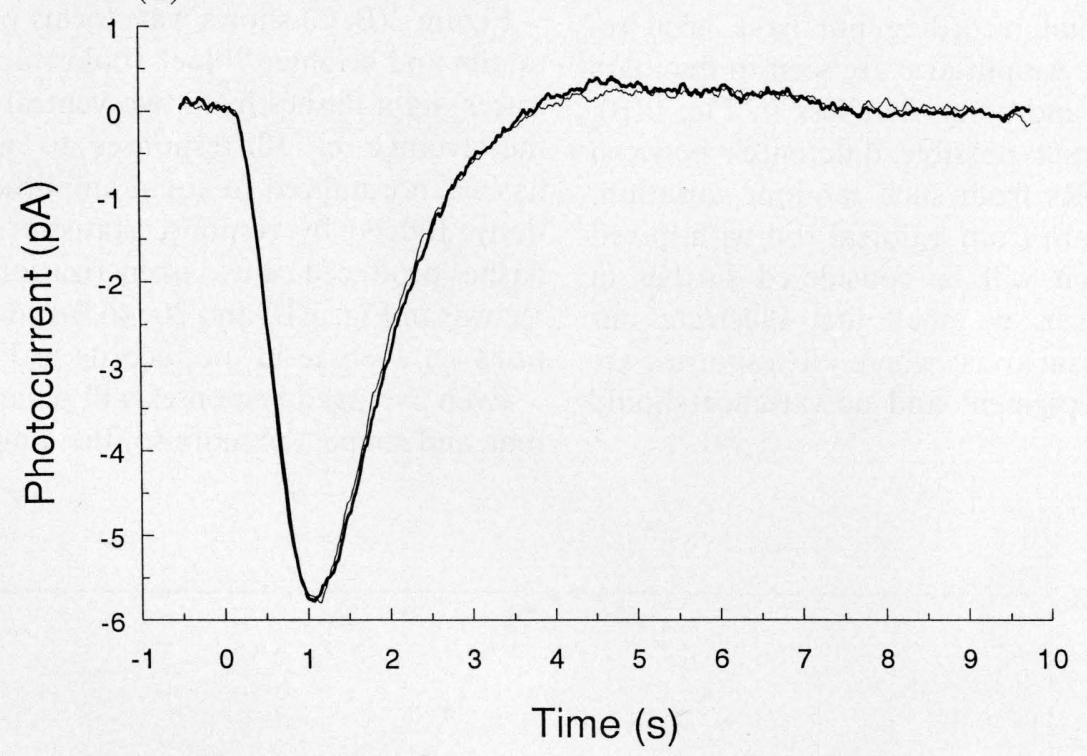

FIGURE 3. (A) Absorption spectrum averaged from the spectra of 27 single rods from one small piece of retina just dorsal to the optic disk (dots). The interrupted curve is the nomogram for rhodopsin ${ }_{502}$. The full-drawn curve, which gives the best fit, is a $98+2 \%$ sum of nomograms for rhodopsin $\operatorname{so2}_{502}$ and porphyropsin ${ }_{527}$. (B, C) Averaged photoresponses from two rods from the ventral retina $[(B)$ and $(C)$ from different rods]. Each trace is the average of 10 responses. Bold traces mark $465 \mathrm{~nm}$ responses and thin traces $608 \mathrm{~nm}$ responses. Flash intensities estimated from response statistics were $c a 2$ isomerizations/flash in (B) and $18(608 \mathrm{~nm})$ and $20(465 \mathrm{~nm})$ isomerizations/flash in $(\mathrm{C})$. The responses have been scaled to equal amplitude, which entailed multiplication of the "red" response by 0.64 in (B) and by 0.9 in (C).

(1) Correction for changes in the total lightsensitive current. If the amplitude of saturated responses, $a_{\max }$, changed during experiments, responses were scaled by factors $a_{\mathrm{MAX}} / a_{\max }(t)$, where $a_{\mathrm{MAX}}$ is the saturating amplitude in the beginning of the experiment and $a_{\max }(t)$ was obtained by linear interpolation between consecutive readings throughout the experiment.

(2) Correction for saturating response compression. As half-saturating intensities were typically $c a 10-20$ isomerizations, the saturating non-linearity of the rod could cause some compression of even fairly "small" esponses. The correction was based on Michaelis-type saturation:

$$
a^{\prime}=a \times a_{\max } /\left(a_{\max }-a\right)
$$

where $a$ is the recorded and $a^{\prime}$ the corrected response amplitude.

These corrections are not particularly critical for the analysis of univariance, as we took care to adjust the intensities of blue and red test flashes to produce responses of approximately the same size. Thus the two sets of responses would be affected equally by the saturating nonlinearity. 


\section{RESULTS}

Variation between single responses: photon fluctuations and intrinsic variability

When a rod is repeatedly presented with nominally identical flashes, individual responses vary in amplitude and shape for at least three reasons. First, the numbers of photoisomerizations actually produced on each trial vary around a mean value according to Poisson statistics. Second, the phototransduction processes in the rod are intrinsically variable, "noisy" (Baylor, Matthews \& Yau, 1980). Third, both the amplitude and shape of the SQR vary systematically depending on the site of photon absorption along the outer segment (Schnapf, 1983), which with full-field, transverse illumination (as here) will vary randomly.

Figure 2 illustrates the variation in series of responses to nominally identical stimuli: dim flashes in Fig. 2(A) and about four times stronger flashes in Fig. 2(B). In both panels, the responses to 465 and $608 \mathrm{~nm}$ were interleaved in the actual recording but have been regrouped for the figure. Amplitudes are seen to fluctuate strongly in Fig. 2(A) and somewhat less in Fig. 2(B).

Our task is to extract possible differences between "red" and "blue" SQRs from such random variation. The records in Fig. 2 are from a dorsal rod with mixed pigment content, which will be considered further in Figs 4 and 5. However, we shall first illustrate our procedures on rhodopsin rods, where all responses are initiated by the same pigment and univariance should certainly hold.

\section{Rhodopsin rods}

The analysis involves microspectrophotometrical determination of pigment composition and comparison of the amplitudes and waveforms of 465 and $608 \mathrm{~nm}$ responses. Representative data for (virtually) pure rhodopsin rods are shown in Fig. 3.

Figure 3(A) shows the average of absorbance spectra from 27 rods in a small piece of dorsal retina. Two nomograms are drawn near the experi-mental points: the interrupted line is that for pure rhodopsin while the full-drawn line corresponds to a $98+2 \%$ mixture of rhodopsin and porphyropsin. The fit of the latter is perceptibly better, showing that even such a small admixture of porphyropsin is detectable (although functionally negligible for our purposes: see Fig. 1). Thus, the analysis of pigment content can resolve differences of a few percent, although the accuracy of the values will of course ultimately depend on the applicability of the nomograms we use.

Figure 3(B, C) shows waveforms of current responses to dim and brighter "blue" (bold traces) and "red" (thin traces) light flashes from two ventral rods. Each trace is the average of 10 responses to nominally identical flashes, normalized to equal amplitudes (see figure caption). Judged by response statistics [equation (1)], the flashes produced $c a$ two isomerizations on average in the records in Fig. 3(B) and 20 (465) and 18 (608) isomerizations on average in the records in Fig. 3(C).

Even averaged responses will of course vary in amplitude and shape, the more so, the dimmer the flashes and

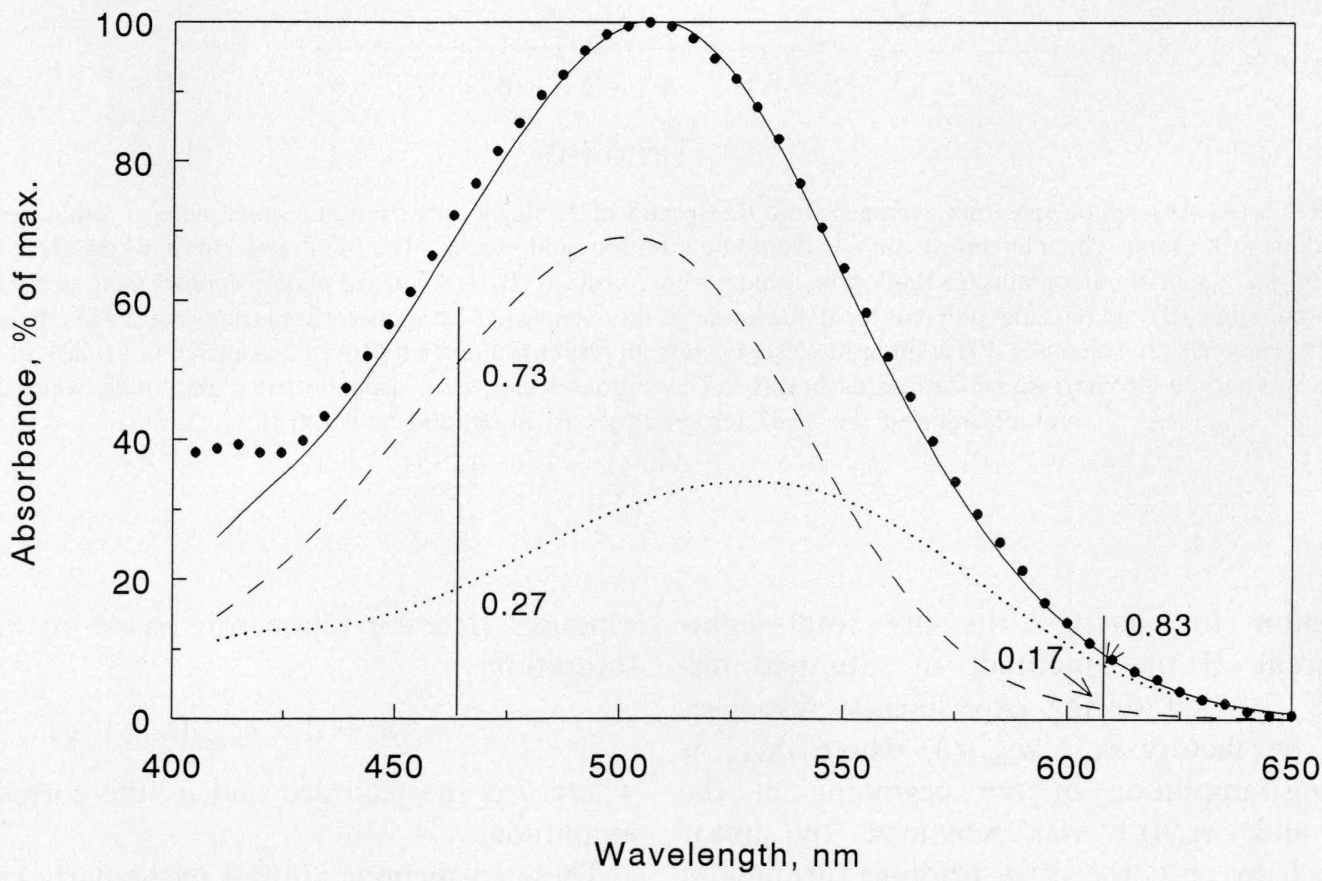

FIGURE 4. Absorption spectrum averaged from spectra of 41 single rods in one small piece of dorsal retina (solid circles). The full-drawn curve fitted to the data is a $67+33 \%$ sum of rhodopsin $_{502}$ and porphyropsin ${ }_{527}$ nomograms (these components are shown as interrupted and dotted curves). The vertical lines illustrate that $73 \%$ of all isomerizations by $465 \mathrm{~nm}$ photons will occur in rhodopsin and $27 \%$ in porphyropsin, whereas $83 \%$ of all isomerizations by $608 \mathrm{~nm}$ photons will be in porphyropsin and only $17 \%$ in rhodopsin. The records in Figs 2 and 5 are from a rod from the same piece of retina as this absorption spectrum. 
(A)

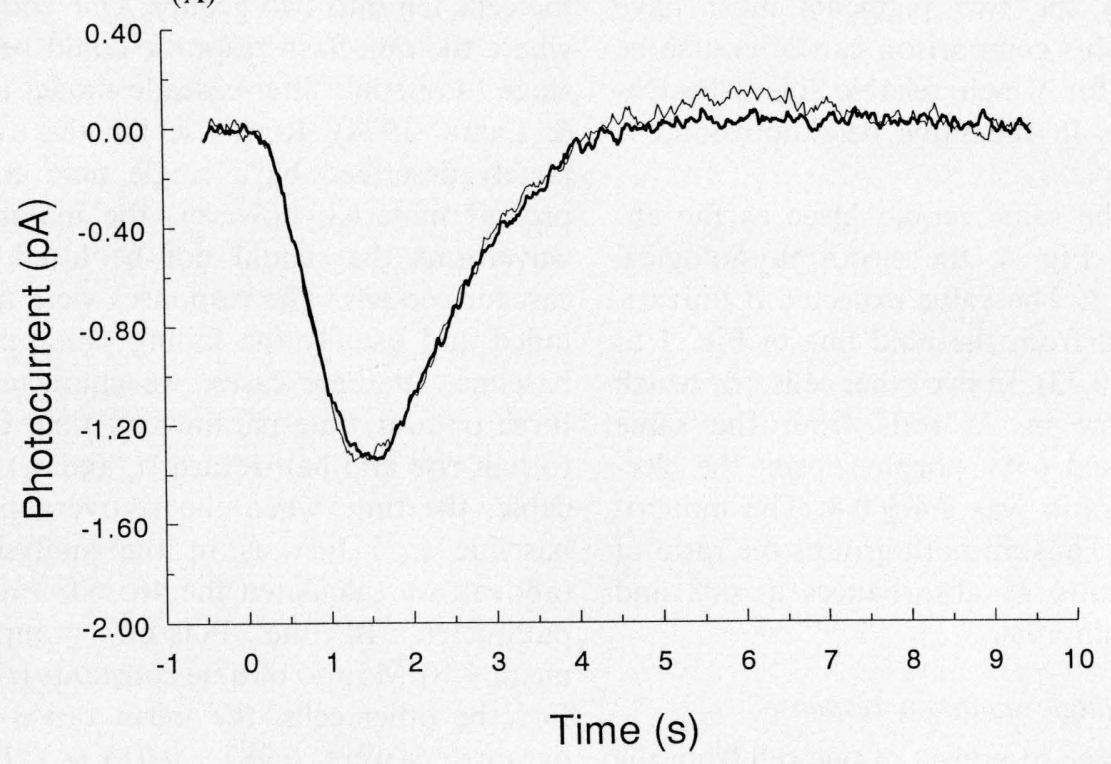

(B)

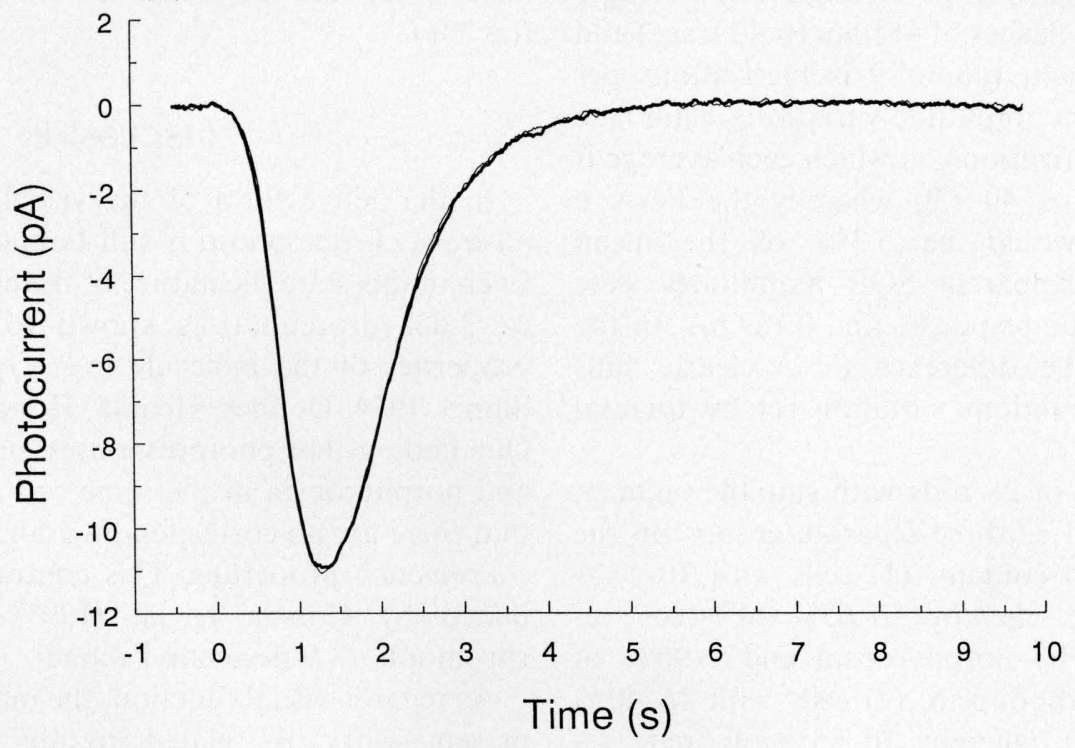

FIGURE 5. Responses from a dorsal rod of mixed pigment content (same cell as in Fig. 2). (A) Averages of 40 responses to $465 \mathrm{~nm}$ (bold trace) and $608 \mathrm{~nm}$ (thin trace) flashes; mean intensity ca 9 isomerizations per flash. (B) Averages of 10 responses to stronger flashes of $465 \mathrm{~nm}$ (bold trace) and $608 \mathrm{~nm}$ (thin trace) light. The responses have not been scaled in this figure. As flashes in (B) were only ca four times stronger than in (A), the responses in (B) appear to be too large. The reason is that they were collected near the beginning of the long experiment over which the amplitude of saturated responses fell from $24 \mathrm{pA}$ to a final value of $7 \mathrm{pA}$.

the fewer the responses summed. [The averages in Fig. 3(B), for example, "contain" a total number of $c a$ $10 \times 2=20$ isomerizations, so the standard deviation due to quantal fluctuations alone will exceed $\pm 20 \%$.] In the figure, the responses have been scaled to equal amplitude to facilitate comparison of response kinetics. The larger responses, which are less sensitive to the rod's intrinsic variation then become virtually indistinguishable [Fig. 3(C)], while clearer differences remain in the waveform of the small responses [Fig. 3(B)]. As "blue" and "red" SQRs in pure rhodopsin rods should in principle be identical, these provide a kind of visual yardstick by which to judge differences in rods with mixed pigment content.
Absorbance and spectral sensitivity in rods with mixed pigment content

The absorbance spectrum in Fig. 4 (solid circles) was averaged from the spectra of 41 rods in one small piece of dorsal retina. The nomogram fit indicates $33 \%$ porphyropsin and $67 \%$ rhodopsin, near-ideal for differentiation (cf. Fig. 1). Also shown are the component rhodopsin and porphyropsin spectra.

A straightforward test for univariance of response amplitudes is to compare the electrophysiologically measured spectral sensitivity with the absorbance spectrum. If the two give the same result in rods suitable for rhodopsin/porphyropsin differentiation, the quantal 
responses initiated by the two pigments must have the same amplitude. This comparison can of course be applied only to cells for which reliable microspectrophotometry from rods in the same neighbourhood is available.

In five cells from the same retinal piece as the absorbance spectrum in Fig. 4, the electrophysiological $\mathrm{R} / \mathrm{B}$ ratio was $4.5 \pm 0.3$. The value expected if univariance holds is 4.4 (read from the bold line in Fig. 1 at porphyropsin fraction 0.33 ). In five other cells, for which microspectrophotometry in 23 rods from the same neighbourhood indicated $65 \%$ porphyropsin, the electrophysiological $\mathrm{R} / \mathrm{B}$ ratio was $7.4 \pm 0.8$. The univariance prediction is 8.0. Thus, in both groups the ratio of sensitivities and the ratio of absorbances at 608 and $465 \mathrm{~nm}$ coincided within $10 \%$.

\section{The amplitude of the single-quantum response}

Figure 5 shows average responses of one cell from the same piece of retina that yielded the spectrum in Fig. 4 (the same cell as in Fig. 2). Figure 5(A) shows averages of responses to $40 \mathrm{dim}$ flashes of $465 \mathrm{~nm}$ (bold trace) and $608 \mathrm{~nm}$ (thin trace) light (about 9 isomerizations per flash as estimated from amplitude variation). Thus, the total number of isomerizations on which each average is based is about $350(\mathrm{ca} 40 \times 9)$ whereby the Poisson standard deviation would be $5.3 \%$ of the mean $(=100 \times \sqrt{350} / 350)$. Apparent SQR amplitudes were $0.195 \mathrm{pA}$ for the $465 \mathrm{~nm}$ responses and $0.189 \mathrm{pA}$ for the $608 \mathrm{~nm}$ responses. The difference $(3 \%)$ clearly falls within the range of random variation set by quantal fluctuations alone.

The whole material of 28 rods with suitable pigment mixtures was analysed as three separate groups on the basis of porphyropsin content: (1) cells with $10-16 \%$ porphyropsin $(n=5)$, whereby $50-65 \%$ of "red" responses are initiated by porphyropsin and $>90 \%$ of "blue" responses by rhodopsin, (2) cells with $16-40 \%$ porphyropsin $(n=15)$, whereby $70-85 \%$ of "red" responses are initiated by porphyropsin and $72-85 \%$ of "blue" responses by rhodopsin, and (3) cells with $40-65 \%$ porphyropsin $(n=8)$, whereby $86->95 \%$ of "red" responses are initiated by porphyropsin and $42-67 \%$ of "blue" responses by rhodopsin.

For each cell, the ratio of SQR amplitudes under 465 and $608 \mathrm{~nm}$ stimulation was estimated from response statistics. The mean \pm SEM $465 / 608$ amplitude ratios obtained for the three groups were (1) $1.05 \pm 0.27$, (2) $0.999 \pm 0.114$ and (3) $0.81 \pm 0.17$. Even the largest deviation from the univariance prediction 1.00 , that of group (3) which amounts to $1.1 \mathrm{SEMs}$, is not significant $(P>0.3$ on a $t$-distribution with d.f. $=7)$. The conclusion for all groups is that the amplitudes of SQRs initiated by rhodopsin and porphyropsin do not differ statistically significantly.

\section{The shape of the single-quantum response}

To compare response kinetics more quantitatively than by visual inspection, characteristic temporal parameters were analysed. As regards response waveform, the cells fell into two groups. One comprised eight cells where the dim-flash response could be fitted by a fourstage "Poisson" filter-cascade model (Baylor, Hodgkin \& Lamb, 1974). Response kinetics can then be completely described by a single time constant $\tau$. In the present material, however, the majority of cells gave waveforms that could not be fitted by simple filtercascade models. The responses were more sharply curtailed and usually the falling phase even overshot the baseline. In these cases, we characterized kinetics by three or four time parameters: time to peak $\left(t_{\mathrm{p}}\right)$, time to half-rise and half-return $\left(t_{\mathrm{r}}\right.$ and $\left.t_{\mathrm{d}}\right)$, and when applicable, the time when the recovery phase crossed the baseline $\left(t_{0}\right)$. Just as in the analysis of amplitudes (above), we calculated the $465 / 608$ ratios for each time parameter. In the Poisson group of cells, the mean \pm SEM ratio of time constants $(\tau)$ was $0.99 \pm 0.02$. For the other cells, the mean ratios of the four time parameters were: $0.987 \pm 0.015\left(t_{\mathrm{r}}\right), 1.007 \pm 0.011\left(t_{\mathrm{p}}\right)$, $1.018 \pm 0.012\left(t_{\mathrm{d}}\right)$ and $1.034 \pm 0.027\left(t_{0}\right)$. Thus, univariance of response shape held to within measurement error (ca $3 \%$ ).

\section{DISCUSSION}

In the active form of the visual pigment (MII) the all-trans chromophore is still bound to its original site. Even in this state the nature of the chromophore (retinal or 3-dehydroretinal) is known to affect the spectral properties of the molecule (e.g. Gyllenberg, Reuter \& Sippel, 1974; Donner, Hemilä, Hongell \& Reuter, 1974). Our finding that photoresponses initiated by rhodopsin and porphyropsin in the same rod are identical implies that there are no corresponding differences in the phototransduction properties. This contrasts with results obtained by Corson et al. (1991) with the artificial chromophore 9-desmethyl retinal.

As regards visual function, the main conclusion of the present work is related to the relative merits of rhodopsin and porphyropsin from a signal/noise viewpoint. We have previously considered the idea, essentially due to Barlow (1957), that the selection between the two is determined by two pressures that will often appear as opposite: (1) the pigment should be efficient in catching quanta in the particular light environment of the animal; (2) it should be thermally stable to minimize false "photon-like" events due to spontaneous isomerizations. The prevalence of porphyropsin in fresh-water environments (see e.g. Bridges, 1970) that are comparatively "yellowish" is qualitatively consistent with the difference in quantum catch. Similarly, the downwardslooking dorsal (porphyropsin) field in the retina of the water-loving bull-frog will most often look at underwater objects (whereas the ventral rhodopsin field looks up into the air). If quantum catch alone were important, however, porphyropsin would be superior to rhodopsin also in many other (terrestrial) light environments where it is not in fact used. We have shown that in dim light a possible photon-catching advantage of porphyropsin would be largely offset by its relative "noisiness". The 
rate of spontaneous photon-like events in porphyropsin rods is at least eight times higher than in rhodopsin rods calculated per molecule of visual pigment. As a result, porphyropsin may become really advantageous only when there is so much light that statistical fluctuation in the numbers of photons absorbed becomes a more significant source of variability than the spontaneous noise events (Donner et al., 1990).

If photon fluctuation and randomly occurring photonlike events constitute the dominant noise components, the size and shape of the SQR is inconsequential for signal/noise, since real and false events will scale equally anyway. However, there are other types of noise in the retina: "continuous" transduction noise in the rods themselves (Baylor et al., 1980) and more proximal neural noise. In relation to these, the size and shape of the SQR could be important - e.g. a large response at the input stage is less liable to be swamped by proximal noise. In this respect, the present work shows that there is no additional bonus nor cost associated with the use of porphyropsin rather than rhodopsin.

\section{REFERENCES}

Barlow, H. B. (1957). Purkinje shift and retinal noise. Nature, 179, 255-256.

Baylor, D. A., Hodgkin, A. L. \& Lamb, T. D. (1974). The electrical response of turtle cones to flashes and steps of light. Journal of Physiology, 242, 685-727.

Baylor, D. A., Lamb, T. D. \& Yau, K.-W. (1979a). The membrane current of single rod outer segments. Journal of Physiology, 288, 589-611.

Baylor, D. A., Lamb, T. D. \& Yau, K.-W. (1979b). Responses of retinal rods to single photons. Journal of Physiology, 288, 613-634.

Baylor, D. A., Matthews, G. \& Yau, K.-W. (1980). Two components of electrical dark noise in toad retinal rod outer segments. Journal of Physiology, 309, 591-621.

Bridges, C. D. B. (1970). The rhodopsin-porphyropsin visual system. In Dartnall, H. J. A. (Ed.), Handbook of sensory physiology, Vol. VII, Part 1A, the photochemistry of vision (pp. 417-480). Berlin: Springer.

Corson, D. W., Derguini, F., Nakanishi, K., Crouch, R. K., Macnichol, E. F. \& Cornwall, M. C. (1991). Relief of bleaching adaptation and induction of wavelength dependent response shapes by 9 desmethyl retinal in rods. Biophysical Journal, 59, 408a.

Crescitelli, F. (1958). The natural history of visual pigments. Annals of the New York Academy of Sciences, 74, 230-255.

Crescitelli, F. (1990). The natural history of visual pigments. Progress in Retinal Research, 11, 1-32.
Dartnall, H. J. A. \& Lythgoe, J. N. (1965). The spectral clustering of visual pigments. Vision Research, 5, 81-100.

Dawis, S. M. (1981). Polynomial expression of pigment nomograms. Vision Research, 21, 1427-1430.

Donner, K., Firsov, M. L. \& Govardovskii, V. I. (1990). The frequency of isomerization-like 'dark' events in rhodopsin and porphyropsin rods of the bull-frog retina. Journal of Physiology, 428, 673-692.

Donner, K. O., Hemilä, S., Hongell, K. \& Reuter, T. (1974). Longlived photoproducts of porphyropsin in the retina of the Crucian carp. Vision Research, 14, 1359-1370.

Firsov, M. L. \& Govardovskii, V. I. (1990). Dark noise of visual pigments with different absorption maxima. Sensornye Sistemy, 4, 25-34 (in Russian).

Govardovskii, V. I. \& Zueva, L. V. (1988). A simple, high-sensitive recording microspectrophotometer. Tsitologiya, 30, 499-502 (in Russian).

Gyllenberg, G., Reuter, T. \& Sippel, H. (1974). Long-lived photoproducts of rhodopsin in the retina of the frog. Vision Research, 14, 1349-1357.

MacNichol, E. F. Jr (1986). A unifying presentation of photopigment spectra. Vision Research, 26, 1543-1556.

Makino, M., Kuzuo, N. \& Suzuki, T. (1983). Seasonal variation of the vitamin $\mathrm{A}_{2}$-based visual pigment in the retina of the adult bullfrog, Rana catesbeiana. Vision Research, 23, 199-204.

Maksimov, V. V. (1988). Approximation of visual pigment absorbance spectra. Sensornye Sistemy, 2, 3-9 (in Russian).

Mansfield, R. J. W. (1985). Primate photopigments and cone mechanisms. In Fein, A. \& Levine, J. S. (Eds), The visual system (pp. 89-106). New York: Liss.

Naka, K.-I. \& Rushton, W. A. H. (1966). An attempt to analyse colour reception by electrophysiology. Journal of Physiology, 185, 556-586.

Partridge, J. C. \& de Grip, W. J. (1991). A new template for rhodopsin (vitamin $\mathrm{A}_{1}$ based) visual pigments. Vision Research, 31, 619-630.

Pugh, E. N. Jr \& Lamb, T. D. (1990). Cyclic GMP and calcium: The internal messengers of excitation and adaptation in vertebrate photoreceptors. Vision Research, 30, 1923-1948.

Reuter, T. E., White, R. H. \& Wald, G. (1971) Rhodopsin and porphyropsin fields in the adult bullfrog retina. Journal of General Physiology, 58, 351-371.

Schnapf, J. L. (1983). Dependence of the single-photon response on longitudinal position of absorption in toad rod outer segments. Journal of Physiology, 343, 147-159.

Semple-Rowland, S. L. \& Goldstein, B. E. (1981). Segregation of vitamin $A_{1}$ and vitamin $A_{2}$ cone pigments in the bullfrog retina. Vision Research, 21, 825-828.

Tsin, A. T. C. \& Beatty, D. D. (1980). Visual pigments and vitamins $\mathrm{A}$ in the adult bullfrog. Experimental Eye Research, 30, 143-153.

Acknowledgements - We wish to thank Professor Tom Reuter for inspiring discussions. This work formed part of joint project No. 22a of the Russian Academy of Sciences and the Academy of Finland. It was further supported by the Academy of Finland (01/1011872), the Sigrid Jusélius Foundation and the Oskar Öflund Foundation. 